\title{
WeChat-based teaching for an immersion cultural exchange program - a case study in CFL
}

Xiaoqin Huang (D

\author{
Correspondence: Huang.xiaoqin@ \\ hotmail.com \\ Department of Applied Foreign \\ Languages, Chengdu Neusoft \\ University, Chengdu, Sichuan, China
}

\begin{abstract}
This paper reports on an exploratory study investigating WeChat-based learning efficiency in teaching Chinese as a foreign language (CFL). Generated from the Connectivism theory, this study aims at exploring the features of mobile-assisted teaching in an immersion CFL exchange program with the social communication app WeChat as a case in point. WeChat is a multimedia instant messenger application in China. In this two-year-long study, an action study was applied. A total of ten learners of elementary Chinese were involved. The participants received and submitted the assignment via WeChat and were asked to review language points on the weekly course posts. Their posts on WeChat Moments were recorded and then reconstructed into language learning content. This study adopted a mixed-method design. Data were collected through the course learning outcomes, an end-of-semester questionnaire, and follow-up interviews. The results show a positive reception from the learners on WeChat-based learning. This study observes (1) students' evident linguistic development in the spontaneous expression on WeChat; (2) a substantial change toward learning with social media; (3) conscious and unconscious language input and output. Language learners reported that using WeChat (1) provided them with a better opportunity to communicate with native speakers; (2) enabled them to integrate knowledge into life; (3) helped them review class content and share thoughts more efficiently; (4) offered them a long-term supportive Chinese language community.

However, the results also indicate that (1) personal preference to social media should be taken into consideration; (2) WeChat fails to foster autonomous learning in the students; (3) students' participation and motivation declined towards the end of the program. In retrospect, monitoring and supervising WeChat usage when necessary, assigning the topics of the posts, and inspiring interest in learning language by solving real-life problems are recommended.
\end{abstract}

Keywords: CFL, Mobile learning, Chinese as a foreign language, WeChat, Exchange program, Immersion

\section{Introduction}

With the popularity of mobile devices and Internet technology, digital learning has become a novel strategy, complementary to formal learning (Burston 2013). Mobile learning refers to using mobile devices and wireless network technology to obtain educational information, resources, and services so that people can be unfettered by time and

(c) The Author(s). 2019 Open Access This article is distributed under the terms of the Creative Commons Attribution 4.0 International License (http://creativecommons.org/licenses/by/4.0/), which permits unrestricted use, distribution, and reproduction in any medium, provided you give appropriate credit to the original author(s) and the source, provide a link to the Creative Commons license, and indicate if changes were made. 
space. Ubiquitous availability is a key feature. It also has characteristics of mobility, network, personalization, and so on (Burston 2015; Chinnery 2006; Kukulska-Hulme 2009). These advantages are popular in Mobile-Assisted Language Learning (MALL).

Under such circumstances, it is highly possible to employ mobile-app-assisted language teaching to enrich foreign language courses (Burston 2015; Chinnery 2006). With the Connectivism theory framework, this study designs a CFL course with the aid of WeChat and assesses how the language learners perceived WeChat-based learning. Before presenting the theoretical framework, research on WeChat-based language teaching is critically reviewed below.

\section{Related work}

\section{WeChat introduction}

WeChat monthly active users have surpassed one billion, and the average user spends over an hour on the app (Social Media Universe 2018). Leading in the instant communication network social media in China, WeChat stands out with its communication technology, information dissemination, and the third-party software development platform, which makes it must-have software in every phone. Although its users mainly cluster in mainland China, WeChat's influence has spread widely to other parts of the world in Chinese-speaking communities (Jin 2017).

Its popularity comes from its multiple functions with three levels of WeChat usage (Fig. 1). The basic function is the one-on-one chat or the group chat; Users can text, call, leave voice messages, send emojis to each other, or group a cohort of people via 'Chats' features. The second is the Moments sharing. Like news feed on Facebook, users can post texts, photos, and video clips on their Moments. Also, they can get alerts when their posts are replied to or commented on. The third is the platform posting. The platform requires an administrator to set up and post articles to subscribers. In the present study, the researcher has conducted the learning at three levels (Fig. 2), so that

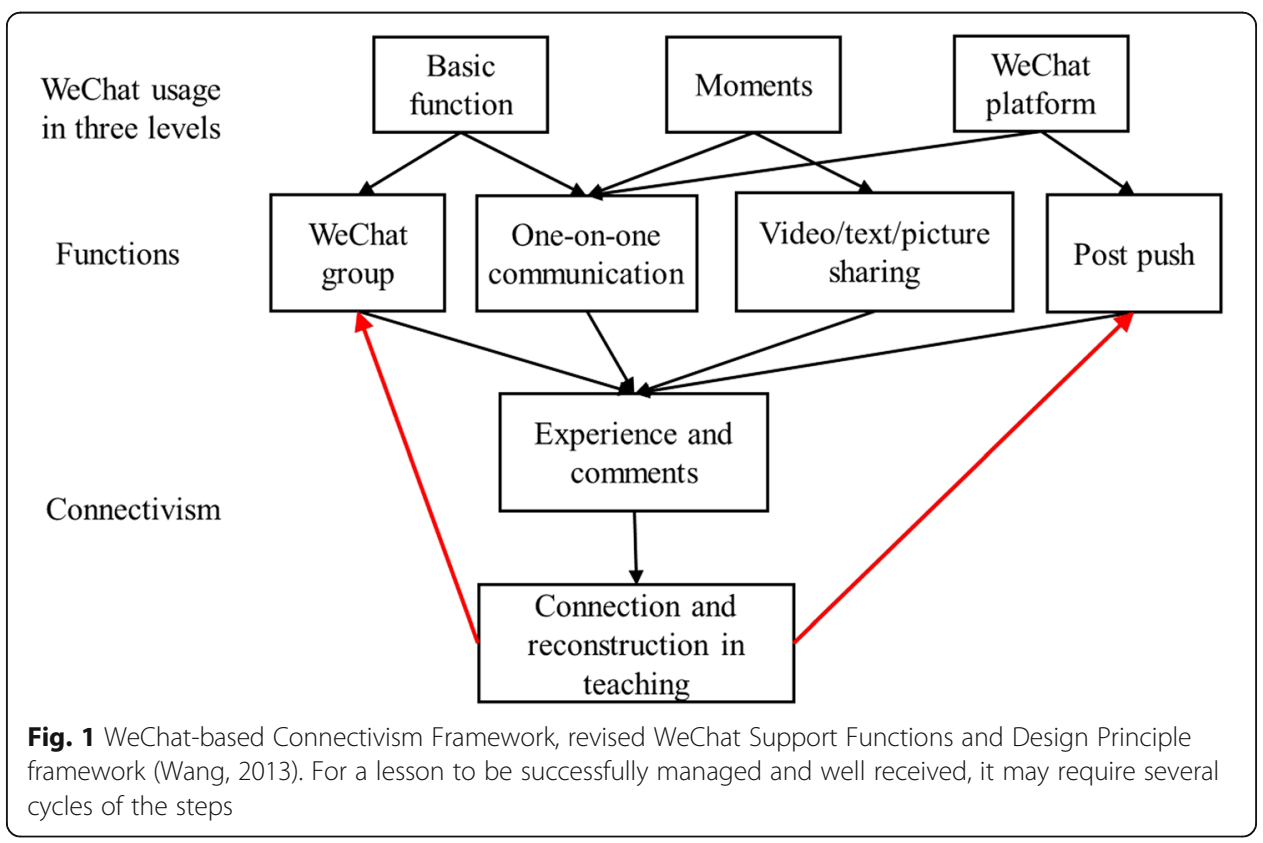


communications can be initiated, teaching materials can be stored, and targeted language can be practiced and discussed (Jin 2017; Wang 2015).

In conclusion, the rationale for employing WeChat as a supplement for the formal course platform has been considered thoroughly: (1) Microenvironment - its functions are adequate for course management and teaching on the three levels. (2) Macroenvironment - the host campus must be Internet capable. Every classroom or building must be equipped with Wi-Fi and Internet ports. Such technical support guarantees smooth communication in WeChat-based learning (Li 2018).

\section{WeChat used in English language learning}

Wuyungaowa (2015) conducted a thirty-eight-day mobile WeChat project. Two classes of second-year university EFL (English as a foreign language), about 60 Chinese students, were involved. In the study, the teacher asked the students to use the textbook vocabulary to communicate as much as possible on WeChat and examined the numbers of students who participated, the number of entries, subjects of chatting and web links that the students shared. She compared the data of participation and effectiveness of the two classes. Results showed the students practiced their listening, writing, speaking, and reading skills to match or accommodate a variety of styles. The weakness of this study was that (1) the students may not have been able to make natural and spontaneous conversations with designated vocabulary; (2) learning groups were too large to inspect so that the teachers' supervision and guidance were hard to guarantee.

\section{WeChat used for bridging CFL learners with Chinese natives}

Jiang and Li (2018) investigated learners' perceptions of a WeChat-embedded Spoken Chinese curriculum. They linked up 17 learners of Chinese in an Australian university with Chinese native speakers via WeChat. The learner-native speaker pairs were to ask and answer five questions of each other in Chinese based on the topics assigned weekly. The Q\&A interactions were recorded on WeChat as voice messages in each WeChat

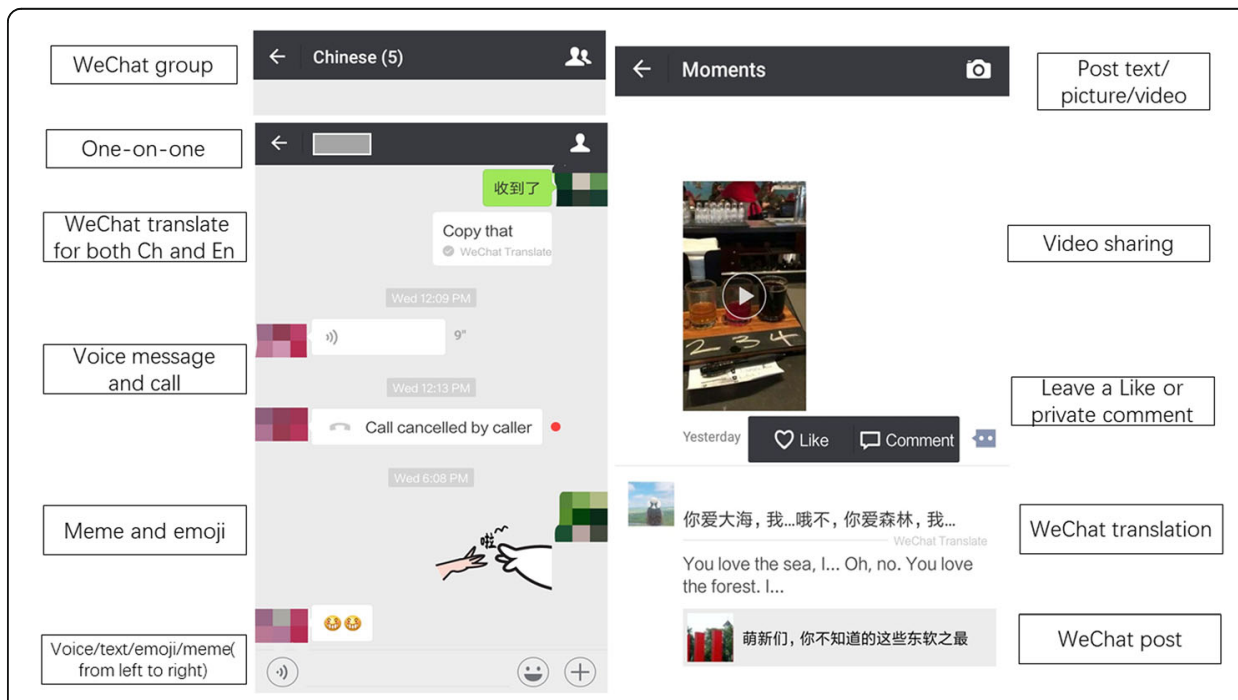

Fig. 2 Screenshots of WeChat Interfaces on the Three Levels 
group, with the instructors as silent listeners. Researchers stated that the WeChat task supplemented classroom learning. However, this study did not fully consider the quality control of the conversations, nor did it analyze the linguistic improvements of the learners, not to mention evaluating the possible influence of the teacher's presence.

Luo and Yang (2016) reported on the implementation of a WeChat project in the Chinese language courses at an American college with students at two proficiency levels of the Chinese language. Results showed that the WeChat project helped them expand the time of learning, improve their Chinese linguistic skills, and enhance language learning motivation. A supportive Chinese language learning community was created as well. However, the weakness of this study is that students from two levels of Chinese, elementary and intermediate, may lead to different degrees of WeChat usage, and the measurement should be tailored accordingly. Besides, the students lack authentic interactions, which may influence the learning effectiveness.

In regard to obtaining authentic linguistic input, Wang et al. (2016) conducted an oral language exchange via WeChat for a semester, linking up learners of Chinese in Australia with native speakers of Chinese in Taiwan. In total, five open-ended mega tasks were assigned consisting of three sub-tasks, namely a recorded speech, a semi-synchronous dialogue, and form-focused feedback. The researchers evaluated the affordances of WeChat in a CFL curriculum under a community of inquiry (CoI) framework. The findings from their study confirmed the establishment of teaching, social and cognitive presences, the three presences of CoI. They concluded that features specific to WeChat supported semisynchronous language exchange.

However, there are some common limitations in the research mentioned above. Firstly, they all focused on either intermediate or advanced level, i.e., 2-year, 1-year, or 3-year CFL background. Secondly, they were long-distance learning, which may entail several problems -1) language learners' contact with native speakers is limited; 2) conversation may be confined to text-based since learners do not share a living or learning context. In other words, the textbook conversation they practiced may be unrelated to their actual lives, which echoes the arguments proposed by the constructivists and connectivists in applied linguistics in that the primary function of language is communication rather than representation. Second language learning occurs most effectively during meaningful interactions (Jiang and Li 2018).

\section{WeChat used in CFL immersion program}

A CFL immersion program is usually characterized by its rigorous monolingual teaching method and the extremely challenging "language pledge" for speaking Chinese only. This teaching style was first invented and developed at Middlebury University in the U.S.A. The results show that adhering to such a program was critical in the students' Chinese learning process (Lou and Yang 2011). Since the policy of Chinese only is more suitable for students who have prior knowledge and proficiency of Chinese, language students at the preliminary level are allowed to use English in the classroom and daily life (Wang and Kirkpatrick 2012).

Jin (2017) applied WeChat in an intensive immersion language abroad program, looking into two undergraduate learners of Chinese language in Shanghai. Participants were asked to fill out weekly surveys to reflect on language learning experience, while the researcher 
conducted participatory observations by collecting screenshots of participants' weekly WeChat activities such as the messages they shared in the WeChat public group and their postings on their respective 'Moments'. The findings showed that compared to other SNSs (Social Networking Services), WeChat provided distinct affordances to the learners at different proficiency levels according to the affordance theory. Notwithstanding the positive result of WeChat application, the number of the subjects was too small, and the researcher was observing instead of participating in WeChat usage and application with the learners. Also, since the study is qualitative research, its language learning efficiency is not addressed, and the possible implementation of the WeChat's other functions is not discussed.

Similarly, Wang (2015)'s research did not include teachers' participation. Wang set up a control team and an experiment team to test the efficiency of learning and score improvement with WeChat aid. The results showed that students regard WeChat as a practical tool for Chinese learning. The attitudes toward WeChat and CFL program imply a positive correlation. She also put forward the three levels of WeChat application, the one-on-one chat, the group chat, and the platform posting \& Moments sharing. However, this study did not include the teacher as an influencing factor, nor did it scientifically analyze the three-level usage in WeChat. For the third level, the platform posting and Moments sharing are two different attributes in usage, access, and audience. Platform posting is an edited post with a specific theme to subscribers, which could be forwarded, liked, and commented on. What is more, the interactions would be public. Moments are text-picture threads published to the owner's friend circle to share one's thoughts on anything, which could only be privately liked and commented on, but not spread. The former is formal and organized in contrast to the latter's immediacy and straightforwardness.

So far, no empirical research has been conducted to examine WeChat-based learning efficiency in an immersion CFL of elementary Chinese setting. To help this cohort of learners in such a setting and to bridge the research gap, the present study endeavors to operate a WeChat-based course for elementary CFL learners by action research and also to examine how the learners perceive the WeChat-based learning. Details of this study and measures to improve the operation of the language course are also discussed in the following sections.

\section{Theoretical framework}

Connectivism is a learning theory for the digital age, a successor to behaviorism, cognitivism, and constructivism (Downes 2012; Siemens 2005). As stated by Siemens, "The act of learning ... is one of creating an external network of nodes-where we connect and form information and knowledge sources. The learning that happens in our heads is an internal network (neural)" (Siemens 2005). His ideas triggered the development of MOOC (Massive Open Online Course) in the 10 years that followed. The concept of the network is prominent in the theory of Connectivism that characterizes knowledge as a flow through a network of humans and non-humans (artifacts). A network comprises connections between entities (nodes), where the nodes can be individuals, groups, systems, fields, ideas, resources, or communities (Bell 2009). In the principles of Connectivism that Siemens set forth, he mentioned the following tips (2005), which are applied in the present study. "Learning is a 
process of connecting specialized nodes or information sources; Learning may reside in non-human appliances; Nurturing and maintaining connections is needed to facilitate continual learning, which emphasized the connections and feedback connections."

The potential of applying the connectivist principles in foreign language education has been discussed (Veselá 2013), but mainly among practicing teachers, sharing their experience on the Internet discussions in blogs, restricting their ideas to a simplified principle of Connectivism as the connection between the learner and diversity of resources. Al-Shehri (2011) illustrates the connectivist principles with the examples of his research of M-learning (Mobile Learning) in EFL at a University. However, that study was not reconstructive and restricted to the use of technologies and social networks.

Foreign language education should be, as maintained by the connectivist principles, based on understanding. It is essential for learners to be able to perceive a language, not as a structure, but as a web of networks linguistically, syntactically, and structurally, and then to comprehend the connections among the nodes within these networks (Siemens 2005). Then, they need to relate the new information to what is already known, changing opinions they had in the past and creating new concepts as new information (Strong and Hutchins 2009). However, whilst the knowledge held by each node is necessary, it is essential to note that within a connectivist-learning network the connections made between nodes should be primarily concerned with the ability to solve problems rather than the specific information held and therefore the network should be filled with contacts and resources rather than solely information (Al-Shehri 2011).

Based on the above theory, one weak point in most previous work applying Connectivism is that although researchers state there are 'connections', they may fail to reconstruct the learning. For example, García et al. (2015) stated there is a lack of means to help low self-motivated or self-regulated students in MOOC forums due to limited information and unstable peer feedback quality. However, the connectivism, in theory, means to extend and reconstruct the learning in and out of the classroom. This point should be highlighted to encourage information flow and to establish standardized assessment systems so as to improve the attention and engagement issues of students with a low degree of self-motivation. As for the present study, the insight would be that in order to enhance CFL learning from the Connectivism perspective, teachers should consider WeChat properties from all three levels for implementing teaching strategies, retrieving and giving feedback, so as to embed them in the learning process.

Drawing on insights and resources provided by previous scholars, the present study designed an action research of a WeChat-based course in a language exchange program at a private college in the autumns of 2017 and 2018. The highlight of this research was to stimulate the learning motivation by combining the learning time in and out of the classroom via the technique of WeChat so as to reconstruct learning. It seeks systematic and linguistic investigation and analysis on how learners perceive the WeChat-based CFL and what improvements can be made for a future course. This paper will first describe the WeChat project and the research design, then investigate the students' perceptions of WeChat-based learning and the effectiveness of incorporating WeChat in elementary CFL by answering the following research questions. 


\section{Research questions}

1. What is the students' perception of the WeChat-based learning method, including discovering whether the method is identified as effective?

2. What are the benefits of using WeChat in elementary CFL learning?

3. What are the disadvantages of using WeChat in elementary CFL, and what possible improvements can be made?

\section{Method}

The study was consecutively conducted from 2017 to 2018, as part of a language exchange program for 12 weeks at a university in China. The host university is located in the suburb and an hour's train away from downtown. Students stayed in a residence hall for international students on campus. During the 12 weeks, each student was responsible for giving lectures for five Spoken English classes as well as attending two or three Chinese language classes (in the year 2018 and 2017 respectively), two Chinese culture classes, and two major classes from Monday to Friday. Spoken English class teaching is as an exchange for the elementary Chinese language class. Students had weekday evenings and most weekends for themselves. Each student was paired with one native language partners, an English major sophomore in the university. The researcher acted as the instructor of the Chinese language course.

\section{WeChat use in the program}

None of the language learners had used WeChat. At the orientation in the host university, the WeChat app was required to be installed on the students' smartphones before the class, and the WeChat group code was given to the learners.

To run a course platform on WeChat, the researcher employed WeChat at three levels as mentioned in Fig. 1. Besides the course-related communication, the WeChat group, and personal chatting (Level 1), the instructor established a WeChat public account to edit, update, and release the weekly posts (Level 3). In the form of videos, audios, pictures, and texts, these posts were to record learners' Chinese language class performance, release a summary of language points, and post exercise for the weekend (Fig. 3). Most importantly, the instructors advised in the First Round but required in the Second Round, the students to post threads in Chinese in the Second Round (Level 2). The instructors recorded the comments and pictures of the students' threads every week and integrated their posts into the classroom teaching and the WeChat weekly posts so as to help enhance the input, reconstruct the language knowledge node, and encourage output. Consent to use the data in this study was obtained from all participants, and all participants' anonymity was protected by numbering the participants, instead of using their real names.

\section{Participants and data collection}

Four learners in 2017 and six in 2018, the ten students were all Caucasian males between18 and 26 years old. Coming from the same Irish university and majoring in the same subject of computer science, they joined this program by applying. Therefore, this study considers that their backgrounds are to be no difference in aspects of intelligence, learning interest, and learning ability. Although the sample size is small, such CFL language programs in 


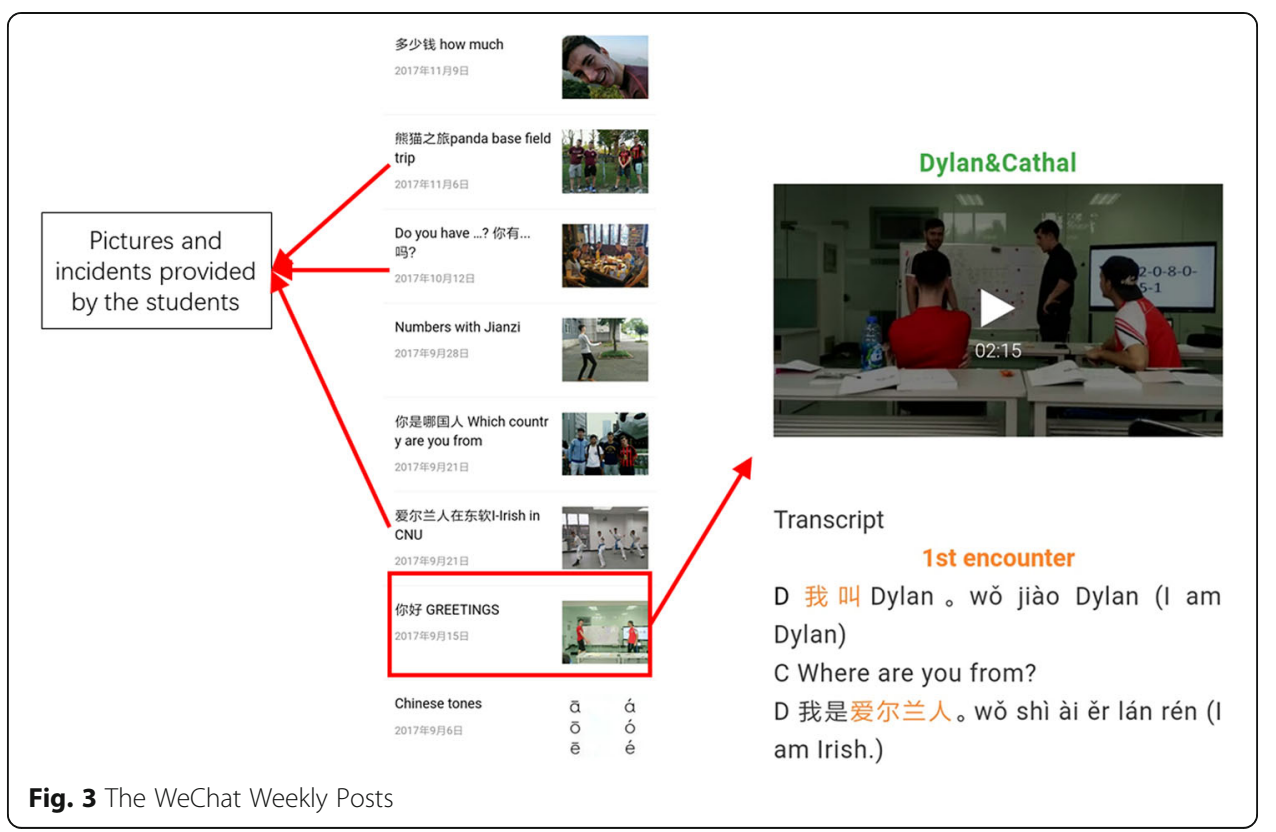

China are not unusual, as they are traditionally organized in a small number of students (Luo and Yang 2016; Jiang and Li 2018; Jin 2017). Besides, the nature of the research has been focused on exploring the possibilities of implementing a social media platform in CFL teaching. The learners in the present study are all Level 1 Chinese learners and have never come to China. So, this study is considered as a typical case study.

\section{Procedure}

In this action research, there are two rounds of investigations, P7-P10 in the First Round of the year 2017 and P1- P6 in the Second Round of the year 2018 respectively. P stands for participants. In the First Round, the researcher did not explicitly require the students to post in Chinese on WeChat, while in the Second Round, the Chinese postings were accounted for performance in the course. The results and findings obtained from Round One were adopted when the researcher designed Round Two, which will be explained in the result section.

Most studies on CFL learning effectiveness are designed to compare and contrast the learning outcomes of the experiment group and the control group (Bot 2014; Lai and Shen 2013; Poole 2015; Xu et al. 2013; Ye 2011). The experiment group is under the research setting, while the control group is under a natural setting. By comparing the learning outcomes of the participants in the two groups, these studies generate the conclusions of their research. In the present study, due to limited participants and resources, the researcher compared the two rounds of research longitudinal chronically in 2 years. The variable in this study is the compulsory usage of WeChat. Under such circumstances, the First Round in 2017 was regarded as the control group, while the Second Round in 2018 as the experiment group.

The learning effectiveness of research question one will be analyzed based on both the learning outcomes and participants' survey on the perception of the WeChat-based pedagogy, which will be discussed in the result section. The learning outcomes are 
assessed from two aspects - formative and summative assessment. The formative assessment consists of participants' performances in class over the program, while the summative assessment is the final examination. Both assessment components account for $50 \%$ of the final course grade.

Two instruments were adopted to investigate how well the learners received the WeChat-based course. At the end of the term, questionnaires and informal interviews were conducted to evaluate WeChat-based language learning.

The questionnaire survey (Additional file 1) includes question types of a 5-point scale, sorting questions, and short answers on how the learners perceived the WeChat-based course. The statements in the questionnaire used in the study were derived from Kim (2014)'s study and revised accordingly. The participants were asked to fill out the survey at the end of the term eliciting their opinions on the use of WeChat in learning Chinese.

The learners graded the practicality of the WeChat program, evaluating their usage of WeChat in three levels of personal or group chats, Moments sharing, and weekly platform posting (Fig. 2). There are four 5-point scale questions, regarding the usefulness of WeChat, with the Cronbach's $\alpha 0.922$ (the highest CITC value (Corrected Item-Total Correlation) 0.881 and lowest 0.733 ; all above 0.7 ), which indicates the reliability of the scale and the consistency among the items (Babbie et al. 2015).

A semi-structured interview (Appendix) was conducted at the end of the term. Conducted in the classroom, each interview was approximately 15-min long. Based on Lee's (2004) study, questions were designed to help the participants recollect what they had gained through WeChat communication and how they perceived their WeChat interactions in terms of language learning. The questionnaire and interview were both focused on learners' experience in learning with WeChat.

\section{Results and analysis}

The ten complete surveys were all valid for data analysis. Both quantitative and qualitative data were collected, together with the instructors' observation throughout the course. With the other factors remaining the same, the only difference for the two rounds is the course requirement on WeChat participation. Compared with the learning performances of the two rounds of research, we see that students' learning outcomes have been effectively improved in terms of formative assessment, and learning motivations have been maintained with the interference of compulsory WeChat usage requirement. Both rounds demonstrated that WeChat was beneficial to language retention, social bonding, and providing feedback to the learners. In the following chapters, research questions will be answered and analyzed.

\section{Learning motivations}

This question was designed to investigate the students' learning motives and outlooks. The major types of motivation for learning are intrinsic and extrinsic. Intrinsic motivation comes from within the student or from factors inherent in the task being performed, while extrinsic motivation comes from sources external to the student and the task, such as praise, recognition, or a system of rewards (Ryan and Deci 2000). As teachers, we hope to promote intrinsic motivation in our students as it encourages life-long learning. While many children are driven 
by external motivators - such as punishment if they get bad grades or rewards if they get good grades - adults are more internally motivated. Adults tend to be more self-directed, internally motivated, and ready to learn. Teachers can draw on concepts of andragogy to increase the effectiveness of their adult education classes (Knowles 1973).

According to Table 1, the degree of motivation for the students learning Chinese is generally high. $60 \%$ of them considered making Chinese friends as the first motive, followed by experiencing Chinese culture and becoming interested in the Chinese language (Table 2). The results imply that the students were driven mostly by internal factors, and they were ready to communicate and learn.

It is worth noticing that although the statistics of learning motivation in Round One is higher than that of Round Two, the participation of the Round One students learning Chinese language decreased more toward the end of the program, while the second-round students had better maintained their performances. The only difference is that Round Two students' WeChat participation of Chinese language was monitored and accounted for in the course. Comparing the performances of the students in the two rounds, we can see intrinsic motivation may be necessary at the start, to prompt them to come to China, while the extrinsic motivation from the teachers plays an essential role in their latter weeks of language learning. Take P4 in the Second Round as an example: this student's motivation was the only one as low as 3 . However, despite 1 week without posting in the middle of the term, P4 produced highly qualitative and informative posts after the teachers' feedback. The details of this participant's attitude change will be explained in the third research question.

At the end of the semester, the researcher interviewed the students on their specific motivational reasons. Students expressed general curiosity and interest in China. Some students demonstrated more thinking: "It (Chinese language) may become an important trait in the world of business (P7)." and "(I learn Chinese is) to be able to make friends easier, because the Chinese students are too shy to speak to us in English (P10).". These understandings were elicited by close observation and personal experience, which immersion learning provides (Lou and Yang 2011). Compared with the research designs of Jiang and Li (2018), Luo and Yang (2016), and Jin (2017), this study was a well-rounded immersion learning. Students not only learned from the classroom, but also extensively experienced a different living environment via online communication. In this case, WeChat plays an essential role in connecting life and learning. It can be seen that such learning provides actual interactions and social situations that lead learners to communicate and think beyond learning. Overall, the students showed on average high intrinsic motivation, and this would be taken into consideration in analyzing the learning effectiveness below.

Table 1 Students' Learning Motivation for the Chinese Language

\begin{tabular}{lllll}
\hline & Minimum & Maximum & Mean & Standard Deviation (SD) \\
\hline Overall Motivation & 3 & 5 & 4.60 & 0.70 \\
Round Two (P1-P6) & 3 & 5 & 4.50 & 0.84 \\
Round One (P7 - P10) & 4 & 5 & 4.75 & 0.50 \\
\hline
\end{tabular}


Table 2 Motivational Reasons

\begin{tabular}{ll}
\hline Reasons & Overall Votes \\
\hline Friends & 6 \\
Culture & 4 \\
Language & 3 \\
\hline
\end{tabular}

\section{Research question 1: efficiency}

The present study considers the efficiency from two aspects - learning outcomes and the survey of learners' perception of the WeChat-based pedagogy. The learning outcomes are comprised of formative assessment and summative assessment. Below is the statistical analysis of participants' learning outcomes (Table 3).

Although the learning motivation results (Table 1) suggested that Round One surpassed Round Two, the learning outcomes showed the opposite, which was in accordance with the observation of their class performance. The course grades of the two rounds are generally the same with Round Two's slightly better than that of Round One's. While the differences in the course grade are not significant, the Standard Deviation of the formative assessment indicates the performance of Round Two students is significantly better than that of the First Round, and the SD of the summative assessment of the Second Round is better than that in the First Round as well. In short, the learning outcomes were generally at the same level, with Round Two significantly or insignificantly better in the formative and summative assessment respectively.

While the learning outcomes of the participants provide a general picture of the learners' learning effectiveness under the setting, the survey offers a more detailed and specific description of their learning with WeChat. To see how they perceived this WeChat-based learning experience, the items of "Please rate your overall experience with the WeChat-based teaching and the learning method" were designed. It turned out the learners had a positive experience in using WeChat to learn Chinese $(\mathrm{M}=3.90, \mathrm{SD}=0.88)$ (Table 4$)$ and recognized that learning with WeChat was productive and effective (Table 4 ).

The SD for the Second Round is slightly higher than the first. It was due to the lowest 2 points from P3. However, while P3 gave the lowest score of 2 on WeChat helpfulness, he was active in social activities, eager to communicate and posted the third-highest posts during his stay. Examining his posts and interview answers, we see that his WeChat use was focused on entertainment rather than the language application, and therefore, he benefited more from social bonding rather than language learning.

To understand how WeChat would be conducive to students' learning, the researcher designed an evaluation of the aspects of WeChat helpfulness. The students gave credits to the different functions of WeChat (Table 5). Applying knowledge into practice by writing, listening, speaking out, posting, and reading was the most useful WeChat function, while submitting homework was the least. The functions of keeping contact, reviewing class

Table 3 Participants' Learning Outcomes

\begin{tabular}{|c|c|c|c|c|c|c|}
\hline & \multicolumn{2}{|c|}{ Formative Assessment } & \multicolumn{2}{|c|}{ Summative Assessment } & \multicolumn{2}{|c|}{ Course Grade } \\
\hline & Mean & SD & Mean & SD & Mean & SD \\
\hline Round Two & 92 & 1.83 & 85 & 9.35 & 89 & 5.30 \\
\hline Round One & 85 & 8.72 & 83 & 13.64 & 84 & 5.58 \\
\hline
\end{tabular}


Table 4 The overall perception of WeChat's productivity in Learning Chinese

\begin{tabular}{lllll}
\hline & Minimum & Maximum & Mean & Standard Deviation \\
\hline Overall perception & 2 & 5 & 3.90 & 0.88 \\
Round Two (P1-P6) & 2 & 5 & 3.83 & 0.98 \\
Round One (P7-P10) & 3 & 5 & 4.00 & 0.82 \\
\hline
\end{tabular}

content, sharing thoughts, and recording life were also approved by learners. From the results, we can see the students' usage of WeChat is interaction-oriented and languagebased.

Apart from its various functions that aid language learning, WeChat is also rich in the language material from the Moments threads and posts, which could provide students with a variety of linguistic input to satisfy different interests and needs, and thus stimulate output. P1 and P5 stated that they were especially interested in the posts about Chinese poetry, while P3 and P4 enjoyed the weekly posts most with the videos of their homework. P2, P4, P7, P8, and P10 declared that the posts of the field trips were the most impressive, and the Chinese vocabulary in the posts was practical. P8 mentioned particularly he felt excited to learn to talk about things that related to him, like drinking beer. He learned the Chinese 'gan bei' for 'bottom-up' in English in class. On that day, he posted a thread entitled "gan bei" on his Moments with a picture of his sharing beers with his Chinese friends. Another time, students P7 and P8 proudly told the teacher that they used the vocabulary to order food, using "zhè gè" (Chinese for "this") and "nà gè" (Chinese for "that"). That is a case where learners consciously applied knowledge into practice, and the sense of achievement was beneficial to their continuous learning.

However, students' Chinese language output was neither persistent nor purposeful. It appears that the learners did not intentionally or systematically apply the class content into usage when chatting with their Chinese friends on WeChat, especially in Round One. This phenomenon may result from an absence in language learning awareness.

Therefore, based on the students' performance in Round One, posting Chinese threads was made compulsory in Round Two. As part of the research, students' posts were intentionally integrated into class content to stimulate the reconstruction of knowledge. In preparing the lessons, the teachers put the pictures and texts of students' postings into the handouts, presentations, sentence examples, and situational dialogues so as to give positive feedback to their engagement on WeChat, encourage language output, and enhance their extrinsic motivation. Learners reported that learning with personal experience was educational and inspirational. Based on the classroom performance and learning results in both

Table 5 Ways that WeChat Helps Chinese Learning

\begin{tabular}{lc}
\hline Functions & Votes \\
\hline Applying knowledge into practice & 7 \\
Keeping contact with friends & 6 \\
Reviewing class content & 6 \\
Sharing thoughts & 5 \\
Recording Chinese life for oneself & 5 \\
Posting homework & 3 \\
\hline
\end{tabular}


rounds, the Second Round of the WeChat-based learning research is substantially more efficient than the First Round with more purposeful language implementation on WeChat. By introducing the materials outside the classroom, the teachers related personal life to language learning, applying the Connectivism theory to bridge the divide between learners' life and classroom learning. This paper further analyzed how WeChat helped students learn the language in question two, and detailed postings are provided.

\section{Research question 2: benefits}

With its multiple functions, WeChat is beneficial to the learner's language learning in several ways, such as linguistic gain, interactive convenience, feedback immediacy, and especially translation friendly to language learners of elementary level. Below, this paper analyzes the benefits in the aspects mentioned above.

Firstly, WeChat enables the learners to apply and learn Chinese language either consciously or unconsciously. In the First Round, there was no requirement for P7-P10 to post Chinese texts. It turns out that students were showing a decline in the participation in either language class activities or WeChat online interactions, with either teacher or their friend. In contrast, the Second Round included WeChat usage as mandatory in the course syllabus. The students were required to post moments in Chinese at least once a week, while the instructors monitored and took records of their posting. These were accounted for $10 \%$ of the Chinese course.

Table 6 shows the statistics of total postings and Chinese posts proportion in the Second Round. Since most of learners' WeChat friends were Chinese students from Spoken English classes, their postings were mostly targeted to such a cohort of the audience. Therefore, their posting languages choice, Chinese or English, implies their attitudes to language and communication.

It is clear that students P1 - P6 generally have used WeChat more frequently, and the proportion of applying Chinese in the Second Round's posts is higher than P7 - P10's in the First Round. In the interview, students were asked about their posting experience with WeChat. P1 stated that with the moments of other Chinese students, he could see how native speakers made sentences with words he has learned. P4 indicated that WeChat posting was helpful to recognize characters. P6 said that with the translation function, he could read Chinese friends' Moments easily and communicate with them freely.

Studying the posting behavior, we see that P1 posted an average amount of posts with a relatively high proportion in Chinese. Noticeably, his English posts were all released in the first month since his arrival, and then he started to use Chinese for the rest. P2 and P3 had posted about 30 Moments respectively, each with a third in Chinese. P4 posted the second least Moments, just about the required amount, while the proportion of his Chinese posts proportion was the highest, followed by P1. His only two posts in English were about bidding his farewell and wishing a happy Christmas. P6 posted the most moments, while P5 followed. Although P6's overall proportion of Chinese posts was relatively small, the theme and Chinese linguistic features of his posts were expanded and improved with time. That is to say, apart from scheduled in-class learning, their language usage was influenced by their extracurricular activities.

To see clearly how the learners developed Chinese linguistic abilities, we take P1 as an example (Table 7) to examine his linguistic improvement. 
Table 6 The Chinese Posts Analysis

\begin{tabular}{|c|c|c|c|c|}
\hline & \multicolumn{4}{|c|}{ Post Analysis } \\
\hline & & Total Posts & $\begin{array}{l}\text { Posts containing Chinese } \\
\text { (including pinyin) }\end{array}$ & Chinese Post Proportion \\
\hline \multirow[t]{6}{*}{ Round Two } & P1 & 21 & 17 & $80.95 \%$ \\
\hline & P2 & 30 & 8 & $26.67 \%$ \\
\hline & P3 & 33 & 11 & $33.33 \%$ \\
\hline & P4 & 14 & 12 & $85.71 \%$ \\
\hline & P5 & 60 & 19 & $31.67 \%$ \\
\hline & P6 & 100 & 14 & $14.00 \%$ \\
\hline \multirow[t]{5}{*}{ Round One } & P7 & 8 & 0 & $0.00 \%$ \\
\hline & P8 & 15 & 3 & $20.00 \%$ \\
\hline & P9 & 23 & 1 & $4.35 \%$ \\
\hline & P10 & 31 & 1 & $3.23 \%$ \\
\hline & & & Mean & $29.99 \%$ \\
\hline
\end{tabular}

Analyzing his posts linguistically, we can see that from September to December, P1 posted 21 Moments with 17 in Chinese. The linguistic features can be observed to be developing along over time. Morphologically, the language of his posting begins with pinyin without tones (the official Romanization system for Chinese), and changes to pinyin-English combination, to pinyin with tones, and to only Chinese characters (simplified and traditional). Along with this improvement, he increased his vocabulary variety, grammar accuracy, and genre types. Not until post No.7, did he post anything besides class content in pinyin- with or without tones and random characters. After post No.8, he began to integrate his life into the postings. From post No.9, the pinyins were complete with tones, but there were some phonetic errors. In addition, P1's Chinese character vocabulary was advancing in the process. By the error analysis, the researcher detected the morphological error in post No. 11, and the syntactic errors in posts No.17 and No.21. The result demonstrated that the error types had been changed from morphologically to syntactically. That is to say, his Chinese progressed. Moreover, in post No.16, he performed a classical style of Chinese writing - using a classical reference in the second person pronoun rather than 'you' - and in No.18, he posted a famous line of a poem in traditional Chinese characters. These phenomena indicated that P1 learned Chinese on his own, attempting to express his ideas about Chinese and interact more with his Chinese friends in the aspects of Chinese language and culture.

Other students, such as P4, P5, and P6, also showed evident progress in Chinese usage. By analyzing the content of their Chinese postings, there was a leap from the classroom content to self-expression 4 weeks after they arrived in China, around the end of September, just like P1. Also, this study observed self-learning output surge after 10 weeks' study, around the beginning of November, since all participants posts texts with vocabularies outside of the textbook on WeChat. The phenomena of group behavior imply that the language input may suffice in assisting a radical improvement in the language output after a process of systematic immersion learning. Despite that the early results may look cautiously promising, much more work is required in the future. 
Table 7 The Chinese posts on P1's WeChat moments

\begin{tabular}{|c|c|c|c|}
\hline No. & Month & P1 & Translation \\
\hline 1 & September & Women shi ai er lan ren & We are the Irish people. \\
\hline 2 & & Ni shi Bolan ren. Ni hen hao & You are Polish. You are very good. \\
\hline 3 & & $\begin{array}{l}\mathrm{Ni} \text { shi Mickey Mouse. }{ }^{2} \text { Shi ni } \\
\text { hao ma? }\end{array}$ & You are Mickey Mouse. How are you? \\
\hline 4 & & English Post No.1 & \\
\hline 5 & & English Post No.2 & \\
\hline 6 & & English Post No.3 & \\
\hline 7 & & jiu bai jiu shi jiu & Nine hundred and ninety-nine \\
\hline 8 & & $\begin{array}{l}\text { aWo yao si shui jiao "I want } \\
\text { four sleep" }\end{array}$ & I want four dumplings. \\
\hline 9 & & $\begin{array}{l}\text { ashéng rì kuài lè Huang } \\
\text { Laoshi }\end{array}$ & Happy birthday, teacher Huang. \\
\hline 10 & October & English Post No.4 & \\
\hline 11 & & 我们 ${ }^{a}$ 系换吃蛋糕 & We like to eat cake. \\
\hline 12 & & 黄老师, 他是谁? & Teacher Huang, who is he? \\
\hline 13 & November & 我喜欢中国画 & I like Chinese paintings. \\
\hline 14 & & 柯博很可怕 & Kebo is very scary. \\
\hline 15 & & 您身体好吗?快点好起来吧 & Are you feeling all right? Get better soon. \\
\hline 16 & & 君莫笑我星期四很累 & Do not laugh. I am very tired on Thursday. \\
\hline 17 & & $\begin{array}{l}\text { a我是坏的手机, 但是上课 } \\
\text { 一下儿 }\end{array}$ & I am a bad phone but going to class for a while. \\
\hline 18 & December & 採菊東籬下 & $\begin{array}{l}\text { (quoting poems in traditional Chinese) Picking } \\
\text { Chrysanthemum at the Eastern Fence }\end{array}$ \\
\hline 19 & & $\begin{array}{l}\text { 在中国的最后一天，我会想 } \\
\text { 你的 }\end{array}$ & The last day in China. I will miss you. \\
\hline 20 & & 直到下一次 & Until next time. \\
\hline 21 & & a它开始的觉得圣诞 & It starts to feel Christmas. \\
\hline
\end{tabular}

Apart from linguistic advancement, WeChat has created a friendly and convenient communication environment for the learners as well as a platform for the language application. Students stated that they made more than 100 friends on WeChat at the end of this program, with $10 \%$ of the friends maintaining constant contact, communicating bilingually. Some learners said the Chinese students would teach them Chinese words and phrases, which the learners would bring to the Chinese class for further learning and discussion. With the help of the translation function in WeChat, the learners would understand their Chinese peers when the language was above their levels or vice versa. This function helps to reduce learning anxiety and encourages interactions at the same time (Krashen 1982, 1985). The learners not only made friends while on campus, but they also built up a long-term relationship with their Chinese friends and teachers after they returned to Ireland. At the end of the programs, the learners P1 to P10 all stated they would continue to use WeChat after they left China. In the following months after the First Round, P7 posted a thread as late as May 2018, a half year later. Similarly, after the Second Round P6 regularly returned to WeChat to post texts and pictures messages in English or Chinese more than 12 times over the next 7 months. According to the previous research, although young students are mostly addicted to the smartphone, their consistent 
attention on a typical app is rare with the rapid shift round the clock (Vacaru et al. 2014). In conclusion, it is obvious WeChat has proved to be a reliable and effective bonding tool, connecting the Chinese to international students, and therefore helped strengthen the learners' language retention and build a life-long learning habit and friendship.

The last point is the function of timely feedback in WeChat's Messaging. A widely cited article contends that assessment for learning would provide information to be used as feedback to modify the teaching and learning activities in which teachers and students are engaged (Black and Wiliam 2010). As teachers check on understanding, they adjust their instruction to keep students on track. Likewise, the students can identify the strengths and weaknesses in their own work, reflect back and on progress, and determine what to do next (Nicol and Macfarlane-Dick 2006). It is evident that quick feedback plays a vital role in learning. As social media software, WeChat enables instantaneous communication for users, which proves efficient language learning. The teachers encouraged interactions on WeChat. When students post their homework via WeChat in forms of pictures, audio or text, the teachers will give specific feedback on the performance of the work in different forms to stimulate a comprehensive development in language learning. Furthermore, the teachers could monitor the group chat and students' moment sharing and respond to their texts and questions within $24 \mathrm{~h}$. That means the group interactions occurred at least three times a week regardless of one-on-one Q\&A, in contrast to their home university's teacherstudent relations in which students have no or few chances talking to their professors on Blackboard, Moodle or other equivalent university platforms. In short, the teacher-student communication on WeChat is productive and functional. The other advantages, such as authentic linguistic resources, multimedia material storage, and the course management platform have also proven to be beneficial to language learning (Luo and Yang 2016).

From the data and observation above, we see that WeChat supports language learning in aspects of authentic language contact, close social bonding, and quick feedback. Its multiple language translation function provides a cushion for language beginners in the immersion program and enables them to switch between languages to adapt to a different language environment. These advantages of WeChat enhanced students' learning and resulted in a better performance in the formative assessment in Round Two compared to Round One.

\section{Research question 3: disadvantages and improvements}

While the benefits of using WeChat are easy to recognize, the disadvantages of applying WeChat in elementary CFL should also be examined. Its strength of flexibility, multifunction, and information abundance as a social media platform could also be its drawbacks of informality, distraction, and information overload for learning.

What is more, if the learners are not social people themselves or tend to decline social media in the first place, they may not be active in using the language to communicate online, in this case, using WeChat. Using P4 as an example (Table 6), we can see his postings were few, and according to his self-report, he did not own any other social media account. His preference for social network indicated that he might not be a good fit in this research setting. During the course, the instructor had closely monitored all the learners' Chinese postings and especially reminded $\mathrm{P} 4$ three times of being late in posting the Chinese homework. In contrast to his low vitality in postings, the quality of his Chinese posting 
is relatively high. It could be detected that his Chinese level has been advanced since he started to use more vocabulary, sentence structures, and manner of speaking in the sentences. His behavior, in this case, echoes his intrinsic low learning motivation but also reflects his interest in Chinese culture and people motivation. When the instructor interviewed him about the change, he shared his new strategy on learning Chinese. He stated that in order to urge himself to interact more on WeChat, he assigned English homework submitted via WeChat for the Chinese students in his spoken English classes. As a result, he combined his work and learning on WeChat, stimulated self-learning motivation, and successfully adapted himself to it by changing his attitude toward this social media-based learning method. It turned out this way of learning was more practical and convenient for a slow learner like him, so as he could learn at his own pace. This incidence indicates the importance of initiative of the learners' initiative to be proactive and cooperative in language learning on social media.

Except for individual preference to social interaction, WeChat's pros could be its cons in certain areas. Since WeChat is a chatting app, it is informal and convenient. People can talk to each other readily. The ideal would be that language learners practice and learn from each other through chat. That is, the Chinese students would practice English with the Irish students, and Irish students would practice Chinese. However, according to the observation and the interview, usually their conversations would end up in English since the Chinese students speak English faster than the language learners' Chinese. Even though the learners speak in Chinese, the speed of information flow in conversations would impel them to choose a faster way to respond instead of taking time in finding the right Chinese words.

Similarly, Li (2018) also stated that without teachers' formal instruction on language practice, students would less likely apply the language on purpose outside the classroom or only confine their language knowledge only to class content or requirements, as demonstrated in the First Round of the present study (Table 6, P7-P10). If the role of teacher or timely feedback is lacking, the learners would easily stray because of distractions on social media, and the connection between learning and using would be uncertain. Thus, it would be difficult for learners to improve their language abilities. Knowledge is not truly acquired, and the relationship between the language and life is disconnected, which leads to low efficiency.

According to the theory, the Connectivism theory serves as a stimulator and a connection between real life and the class contents (Downes 2012; Siemens 2005). In the present study, this connection proves to be a promising role in linguistic acquisition. Evidence can be seen in the learners' linguistic gain (Table 6) and P1's posting example (Table 7). Their postings were mostly derived from their lives and classroom content. When learners were interviewed on the experience of WeChat-based learning, they expressed that WeChat lightened the pressure of learning such a complicated language and inspired them to speak more in Chinese. For example, P3 says, "(WeChat helps me learn Chinese) ... By learning Chinese that you use every day - e.g., ordering food."

Taking the above analysis into consideration, we see that this learning method's advantages outweigh the disadvantages in terms of the learning motivation and the language application as well as the life-long learning attitude and material reconstruction. At the same time, improvements could still be made to counteract the method's 
drawbacks and enhance its strength. Below are the three aspects of improvement suggestions in WeChat-based learning.

Firstly, enhancing the correlation between the language course and learners' WeChat use: The results show that some students did not intentionally pay attention to the language in life and produced less Chinese in WeChat communication. The phenomena could be improved by teachers' giving more specific instructions on WeChat usage. For example, teachers could spare the last $3 \mathrm{~min}$ of class for students to post "What I have learned today" before leaving. Likewise, to increase language input and output, teachers could require students to read the weekly posts and leave comments in Chinese or pinyin. Such activities should be graded in the performative assessment. Furthermore, teachers could assign topics for the weekly homework to urge students to produce themed posts, and in return, it would be applicable for the teachers to examine students' linguistic gain in a more controlled way.

Secondly, the language choice: In the interaction with Chinese peers, the teachers could advise the learners to interact with their Chinese friends bilingually, or in a combination of Chinese and English, and also learners to ask their native friends to speak in Chinese more often or bilingually. Also, the learners could conduct small semi-bilingual videos with native speakers on WeChat as a final project in the course, which should be set advance in the course syllabus.

Thirdly, maintaining and stimulating intrinsic and extrinsic learning motivations: Usually, students would be more active in learning the language at the beginning of the learning and post more threads when in a new environment than following periods when things got familiar (Table 4), or the language difficulty increased. For instance, the first month saw the most Moments posted by P1, while the middle of the term observed zero posts from P4 for a week. To regenerate their interest, the teachers could apply PBL (Problem-based Learning) strategy to set up real-life problems for students to solve. For example, buying a souvenir. This task would involve vocabulary preparation and phonetic, syntactic, or pragmatic practice of interactions. Thus, with various real-life problems, students could be proactive to preview new vocabulary and practice on-site dialogues. What is more, since the learners are exchange students seeking life exploring and culture immersion, activities on culture shock between their home country and China would also be a trigger for language practice. Additionally, comparing their native language to Chinese would also provide more in-depth insights into Chinese learning.

\section{Conclusion}

From the overall investigation, we can see (1) evident linguistic gain of students' spontaneous expression after 4 weeks' study, and self-learning after 10 weeks' study; (2) learners' substantial attitude change toward social media-based language learning via proactive strategies; (3) extensive learning of Chinese through conscious or unconscious language input and output. All the participants showed a positive acceptance of the WeChat application. Through this app, language learners could (1) practice linguistic skills with native speakers; (2) connect knowledge to life; (3) review and share thoughts more efficiently; (4) enjoy a long-term supportive Chinese language community.

However, the results and analysis have also identified some problems: (1) some students demonstrate disinclination to WeChat/social media participation; (2) WeChat did not foster an autonomous learning environment; (3) students' low intrinsic motivations 
towards the end of the program influence their engagement on WeChat. Here suggestions are proposed for improvement. To develop a better environment for WeChat-based learning and research, the instructors should firstly make sure that students are fully informed of the necessity of WeChat usage as part of the immersion language program design, in and out of class; the purpose of the exchange program, and the design of the language course. Then instructors should (1) stimulate self-learning by offering greater diversity and choice of learning material; (2) specify the interactions to be bilingual between the language learners and the native speakers, either compulsorily or voluntarily; (3) stimulate or maintain motivations by PBL methodology.

Likewise, the present study has a few limitations. Firstly, the number of participants $(N=10)$ was small. The future study would be recommended to collect larger samples for a more extended period. Secondly, the participants were all computer science students studying elementary Chinese. It is worth exploring (1) whether students from different majors or backgrounds respond to the WeChat-based learning differently; (2) the incorporation of WeChat into advanced-level Chinese language curriculum; (3) the linguistic leap that may occur after a certain amount of time in the immersion language program. Thirdly, it is hard to compare the data of learning outcomes effectively. This present study applied two-round research in two consecutive years. Although the research has managed to keep the course environment the same, the social environment may be beyond control. Given the specific situation in the immersion CFL programs nowadays where participants and data are both limited, a real comparison of the findings with a synchronized control group is problematic. Finally, many interactions between students and native speakers were private or offline, so that language practice was difficult to track. That indicates a need for future endeavor to find ways to track more detailed data in WeChat-based CFL.

By adopting the Connectivism theory, this exploratory study made empirical contributions to the understanding of the WeChat-based CFL immersion program in qualitative and quantitative as well as practical and academic aspects. It improves previous understandings about WeChat-based pedagogy, while addresses the issues that could be investigated in future research. In sum, WeChat-based course would call for more thoughts on maintaining the motivation, reconstructing materials, and assessing learners' language progress to ensure that the language learning and teaching are systematically and scientifically processed.

\section{Appendix}

The oral interview questions for the language learners at the end of semester

1) Briefly describe your experience with Wechat for this course.

2) Share with me the most valuable and interesting part of Wechat online exchange and why.

3) How do you feel about the teaching strategy that applied your own pictures and experience in class content?

4) Tell me about your experience chatting with native speakers on Wechat. Did you enjoy it and why?

5) What do you think about the topics for Wechat group and Wechat course platform? 
6) Do you think Wechat helped you improve your Chinese? How?

7) Overall, tell me how the experience of Wechat communication exchange changed your perspective on the Chinese culture? Do you have any concerns about Wechat-based course?

\section{Additional file}

Additional file 1: WeChat Questionnaire on the overall Chinese course. (DOCX $18 \mathrm{~kb}$ )

\section{Abbreviations}

CFL: Chinese as a foreign language; CITC: Corrected Item-Total Correlation; EFL: English as a foreign language; MALL: Mobile-Assisted Language Learning; M-learning: Mobile Learning; MOOC: Massive Open Online Course; P: Participant; SNS: Social Networking Services

\section{Acknowledgments}

Not applicable.

\section{Authors' contributions}

The paper and the research on which the paper is based on are of individual effort. The author read and approved the final manuscript.

\section{Funding}

Not applicable.

\section{Availability of data and materials}

Main data and materials are provided upon request.

\section{Competing interests}

The author declares that he/she has no competing interests.

Received: 4 February 2019 Accepted: 30 July 2019

Published online: 09 August 2019

\section{References}

S. Al-Shehri, Connectivism: A new pathway for theorising and promoting mobile language learning. Int. J. Innov. Leadersh. Teach. Humanit. 1(2), 10-13 (2011) https://www.academia.edu/1312249/IJILTH01.02_Saleh_AL-SHEHRI_Connectivism_A_ new_pathway_for_theorising_and_promoting_mobile_language_learning_pp._10-31_. Accessed 4 Sept 2012

E.R. Babbie, W.E. Wagner, J.S. Zaino, Adventures in Social Research: Data Analysis Using IBM SPSS Statistics (Sage, New York, 2015)

Bell, F. (2009). Connectivism: a network theory for teaching and learning in a connected world. http://usir.salford.ac.uk/id/ eprint/2569/1/ConnectivismEdDev.pdf. Accessed 17 June 2018

P. Black, D. Wiliam, Inside the Black box: Raising standards through classroom assessment. Phi Delta Kappan 92(1), 81-90 (2010). https://doi.org/10.1177/003172171009200119

K.-d. Bot, The effectiveness of early foreign language learning in the Netherlands. Stud. Second Lang. Learn. Teach. 4(3), 409-418 (2014). https://doi.org/10.14746/ssllt.2014.4.3.2

J. Burston, Mobile-assisted language learning: A selected annotated bibliography of implementation studies 1994-2012. Lang Learn. Technol. 17(3), 157-224 (2013) https://www.researchgate.net/publication/259079992_Mobile-Assisted_Language_ Learning A selected annotated bibliography of implementation_studies 1994-2012. Accessed 10 Aug 2018

J. Burston, Twenty years of MALL project implementation: A meta-analysis of learning outcomes. ReCALL 27, 4-20 (2015). https://doi.org/10.1017/S0958344014000159

B.H. Chinnery, Going to the MALL: Mobile assisted language learning. Lang. Learn. Technol. 1, 9-16 (2006)

S. Downes, Connectivism and connective knowledge: essays on meaning and learning networks (National Research Council Canada, Ottawa, 2012) Retrieved from http://www.downes.ca/files/books/Connective_Knowledge-19May2012.pdf

B.J. García, G.C. Tenorio, M.S. Ramírez, Self-motivation challenges for student involvement in the open educational movement with MOOC. RUSC. Univ. Knowl. Soc. J. 12(1), 91-103 (2015). https://doi.org/10.7238/rusc.v12i1.2185

W. Jiang, W. Li, Linking up learners of Chinese with native speakers through WeChat in an Australian tertiary CFL curriculum. Asian Pac. J. Second Foreign Lang. Educ. (2018). https://doi.org/10.1186/s40862-018-0056-0

L. Jin, Digital affordances on WeChat: Learning Chinese as a second language. Comput. Assist. Lang. Learn. 31(1-2), 27-52 (2017). https://doi.org/10.1080/09588221.2017.1376687

$\mathrm{H}$. Kim, Effects of using mobile devices in blended learning for English reading comprehension. Multimed. Assist. Lang. Learn. $17(2), 64-85(2014)$

M.S. Knowles, The adult learner: A neglected species (Gulf Publishing Company, Houston, 1973) Revised Edition 1990

S. Krashen, Principles and Practice in Second Language Acquisition (Pergamon Press, Oxford, 1982)

S. Krashen, The Input Hypothesis: Issues and Implications (Longman, Harlow, 1985)

A. Kukulska-Hulme, Will mobile learning change language learning? ReCALL 21(2), 157-165 (2009)

J.-C. Lai, T. Shen, The impact of cultural metaphors on learning effectiveness in English as a foreign language curriculum. Eur. J. Soc. Behav. Sci. 6(3 special issue) (2013). https://doi.org/10.15405/ejsbs.92

L. Lee, Learners' perspectives on networked collaborative interaction with native speakers of Spanish in the US. Lang. Learn. Technol. 8(1), 83-100 (2004) 
Y. Li, Current problems with the prerequisites for flipped classroom teaching---a case study in a university in Northwest China. Smart Learn. Environ. 5(1) (2018). https://doi.org/10.1186/s40561-018-0051-4

K. Lou, T. Yang, in Chinese Language Globalization and Pedagogy (Volume One)-the Ninth International Symposium on Chinese Language Teaching Papers Collection. The impact of American short-term Chinese programs in China on Chinese teaching mode of Middlebury college - based on PIB, CIB and HBA (China Minzu University Press, Beijing, 2011), pp. 210-219

H. Luo, C. Yang, Using WeChat in teaching L2 Chinese: An exploratory study. J. Technol. Chin. Lang. Teach. 7(2), 82-96 (2016) http://www.tclt.us/journal/2016v7n2/luoyang.pdf. Accessed 20 Aug 2018

D.J. Nicol, D. Macfarlane-Dick, Formative assessment and self-regulated learning: A model and seven principles of good feedback practice. Stud. High. Educ. 31(2), 199-218 (2006). https://doi.org/10.1080/03075070600572090

F.J. Poole, in All Graduate Plan B and other Reports. Teaching Chinese as a Foreign Language: A Foreigner's Perspective (2015), p. 457 https://digitalcommons.usu.edu/gradreports/457. Accessed 11 May 2019

R.M. Ryan, E.L. Deci, Self-determination theory and the facilitation of intrinsic motivation, social development, and well-being. Am. Psychol. 55(1), 68-78 (2000). https://doi.org/10.1037/0003-066X.55.1.68

G. Siemens, Connectivism: A learning theory for the digital age. Int. J. Instr. Technol. Dist. Learn. 2(1), 3-10 (2005)

Social Media Universe. (2018). Visualizing the Social Media Universe in 2018. http//wwww.visualcapitalist.com/social-media-universe/. Accessed 17 Aug 2018

K. Strong, H.M. Hutchins, Connectivism: A theory for learning in a world of growing complexity. Int. J. Appl. E-Learning 1(1), 53-67 (2009)

M.A. Vacaru, R.M. Shepherd, J. Sheridan, New Zealand youth and their relationships with Mobile phone technology. Int. J. Ment. Heal. Addict. 12(5), 572-584 (2014). https://doi.org/10.1007/s11469-014-9488-z

K. Veselá, Connectivism in foreign language education. Educ. Lang. Eur./Bildung Sprachen Europa 25(17), 320-325 (2013)

D. Wang, A. Kirkpatrick, Code choice in the Chinese as a foreign language classroom. Multiling. Educ. 2(1), 3 (2012). https:// doi.org/10.1186/2191-5059-2-3

Y. Wang, W.-C. Fang, J. Han, N.-S. Chen, Exploring the affordances of WeChat for facilitating teaching, social and cognitive presence in semi-synchronous language exchange. Australas. J. Educ. Technol. 32(4), 18-37 (2016)

Z. Wang, Weixin pingtai zai yuyan xuexi zhong de yingyong yanjiu (微信平台在语言学习中的应用研究), English translation title: Effects of using WeChat in an experimental CSL program. Open Educ. Res. 21, 113-118 (2015)

Wuyungaowa, Engaging EFL learners through WeChat: A Mobile phone-based EFL learning project in China. Int. HETL Rev. 5(5) (2015) https://www.hetl.org/engaging-efl-learners-through-wechat-a-mobile-phone-based-efllearning-project-in-china. Accessed 10 Aug 2018

Y. Xu, L.Y. Chang, J. Zhang, C.A. Perfetti, Reading, writing, and animation in character learning in Chinese as a foreign language. Foreign Lang. Ann. 46(3), 423-444 (2013). https://doi.org/10.1111/flan.12040

Ye, L. (2011). Teaching and Learning Chinese as a Foreign Language in the United States: To Delay or Not to Delay the Character Introduction. Dissertation, Georgia State University. https://scholarworks.gsu.edu/alesI_diss/21. Accessed 11 May 2019

\section{Publisher's Note}

Springer Nature remains neutral with regard to jurisdictional claims in published maps and institutional affiliations.

\section{Submit your manuscript to a SpringerOpen ${ }^{\circ}$ journal and benefit from:}

- Convenient online submission

Rigorous peer review

- Open access: articles freely available online

- High visibility within the field

- Retaining the copyright to your article

Submit your next manuscript at $\boldsymbol{\nabla}$ springeropen.com 